\title{
A Green Analytical Method Using Polyurethane Foam for The Extraction and Determination of Lauryl Ether Sulfate in Personal Care Hygiene Products
}

\author{
Felipe R. Müzel, ${ }^{a}$ Eduardo L. Rossini ${ }^{\oplus a}$ and Helena R. Pezza ${ }^{\oplus *, a}$ \\ Instituto de Química, Universidade Estadual Paulista Júlio de Mesquita Filho (Unesp), \\ Rua Prof. Francisco Degni, 55, CP 355, 14800-900 Araraquara-SP, Brazil
}

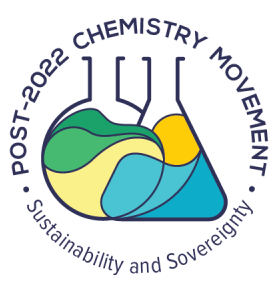

A green methodology was developed for the extraction and determination of lauryl ether sulfate in raw materials and commercial liquid soap and shower gel samples. The method avoids the use of organic solvents, offering a simple, cheap, and safe analysis. The methodology is based on the sorption of an ionic pair consisting of a cationic dye and an anionic surfactant onto polyurethane foam. The experimental variables were optimized by chemometry to obtain the conditions that maximized extraction of the ionic pair. Digital imaging and spectrophotometry were used for quantification in the linear concentration range from 10.0 to $100 \mathrm{mg} \mathrm{L}^{-1}$. The limits of detection and quantification were, respectively, 2.71 and $9.28 \mathrm{mg} \mathrm{L}^{-1}$ for digital imaging, and 1.74 and $5.83 \mathrm{mg} \mathrm{L}^{-1}$ for spectrophotometry. The methods showed good results when applied to commercial samples, with recoveries in the range $96.8-103 \%$.

Keywords: green chemistry, lauryl ether sulfate, anionic surfactant, digital imaging, spectrophotometry

\section{Introduction}

Surfactants are amphiphilic compounds that aggregate to form micelles, resulting in emulsifier activity that enables the solubilization of compounds with low solubility and alteration of the surface tension of solutions used in the production of detergents, cleaning products, and personal hygiene products. ${ }^{1-4}$ For these purposes, anionic surfactants are the class most widely used. ${ }^{5}$ Products derived from anionic surfactants can be produced simply, at low cost, ${ }^{4}$ and have broad application in industry, due to their foaming and cleaning properties. ${ }^{4,6-9}$ Sulfonates and alkyl sulfonates are the most popular anionic surfactants, since they present high detergent activity and have lower environmental impacts, compared to the anionic surfactants used previously. ${ }^{10,11}$

Even using sulfonates and alkyl sulfonates, high levels of discharges of anionic surfactants into aquatic systems lead to alterations of the surface tension, density, and viscosity of water, which negatively affects living organisms, while the formation of high amounts of foam increases the cost of water treatment. ${ }^{12-16} \mathrm{At}$ the present time, the emergence of the COVID-19 (Coronavirus Disease 2019) pandemic

*e-mail: hr.pezza@unesp.br

Editor handled this article: Eduardo Carasek has led to extensive use of liquid soaps or shower gels containing anionic surfactants for hand hygiene. ${ }^{17}$ Given the impacts of these substances, it is important to monitor the concentrations of anionic surfactants arising from the industrial production of cosmetics and hygiene products.

Methods that have been described for the analysis of anionic surfactants include classical volumetric techniques based in the titration of two phases, ${ }^{18,19}$ spectrophotometry, ${ }^{20,21}$ chromatography, ${ }^{22,23}$ and potentiometry. ${ }^{24-26}$ However, many of these methods commonly use an organic solvent, necessitate sample clean-up steps, require expensive equipment, or are laborious. ${ }^{17-25}$ The official method ${ }^{27}$ for the determination of anionic surfactants is based on the reaction between the surfactant and a cationic dye, forming an ionic pair that is extracted into an organic phase (chloroform). This method suffers from many drawbacks, including the use of large volumes of chloroform $(100 \mathrm{~mL}$ for a single analysis), numerous interferences, long analysis times, and difficulty in analyzing colored samples. Consequently, there is the need for simple, low-cost, and clean methods for the determination of anionic surfactants, which comply with the principles of Green Chemistry, ${ }^{28-30}$ replacing chloroform by another extractor.

Polyurethane foam (PUF) has been shown to be a good option as a solid phase for the extraction of different substances, following the principles of Green Chemistry. ${ }^{31-36}$ 
Due to the presence of polar and non-polar groups in its structure, it can interact in different ways with many compounds and reagents, including the ionic pair formed between an anionic surfactant and a cationic dye. PUF is a low-cost material that is stable, widely available commercially, and easy to handle without any need for pretreatments. Consequently, it has broad applications, with the additional advantage that it can be reused for the same extraction process, without loss of efficiency. ${ }^{32,33}$

Specific methodologies have been developed for the analysis of surfactants using commercial or modified polyurethane foams for extraction of the ionic pair formed between methylene blue (MB) and the anionic surfactant sodium dodecylsulfate (SDS). ${ }^{35,36}$ Measurement of the colored species (ionic pair) on the foam surface was performed by diffuse reflectance, with treatment of digital images recorded using a digital scanner ${ }^{35}$ or smartphone. ${ }^{36}$ However, a filtration step was required before detection, ${ }^{35}$ the polyurethane foam was not commercial, ${ }^{36}$ and the methods were only applied for the analysis of water samples. ${ }^{35,36}$ Furthermore, it is usually difficult to obtain reproducible results, due to the effects of particle size and the surface of the PUF on the reflectance phenomena.

It is difficult to determine anionic surfactants in cosmetics or personal hygiene products, due to the complexity of the matrices, which include emulsions, dispersions, solutions, and solids. ${ }^{37}$ To the best of our knowledge, there have been no previous reports on the use of PUF as an extractor applied to cosmetic or personal care product matrices, with detection by spectrophotometry or digital imaging.

The purpose of this work was to develop a clean methodology for determination of anionic surfactants, more specifically lauryl ether sulfate (LES), in personal hygiene and cosmetic products, as well as in the raw materials.

The alternative green analytical method developed in the present work was based on measurement of the color intensity of methylene blue in solution, before and after extraction of the ionic pair formed by MB and LES onto polyurethane foam, with detection by spectrophotometry and digital imaging. Optimization of the experimental variables was carried out using factorial design. The proposed green assay was applied to cosmetics, personal hygiene products, and raw materials.

\section{Experimental}

Apparatus

Absorbance measurements $\left(\lambda_{\max }=665 \mathrm{~nm}\right)$ were performed using a diode array UV-Vis spectrophotometer
(HP 8453A, Hewlett Packard, California, USA) equipped with a $1 \mathrm{~cm}$ path length glass cell. Eppendorf micropipettes (Barkhausenweg, Germany) were used to deliver the smaller volumes of solutions. Magnetic stirrers (Model 114-1, Ethik Technology, São Paulo, Brazil; Model 752, Fisatom, São Paulo, Brazil; Model RH basic, Merse, São Paulo, Brazil) were used to promote the sorption process.

Digital images were acquired using a cell phone equipped with a flash lamp (Iphone SE, Apple, California, USA). The solutions were transferred to the wells of a porcelain plate that was then placed in a homemade box (dimensions $40 \times 15 \times 20 \mathrm{~cm}$ ) for obtaining photographs of the wells. The images were analyzed using free ImageJ software. ${ }^{38}$

\section{Materials, reagents, and solutions}

Ultrapure water (18 M $\Omega \mathrm{cm}$, Milli-Q system, Millipore, Darmstadt, Germany) was used to prepare the solutions and dilutions. Commercial polyurethane foam (type D28 off-white), purchased locally in Araraquara (São Paulo State, Brazil) was cut into pieces $(1.00 \times 2.50 \times 3.75 \mathrm{~cm})$ and was used without any pretreatment.

A stock solution of sodium dodecyl sulfate (SDS, $98.5 \%$, molar mass $=288.37 \mathrm{~g} \mathrm{~mol}^{-1}$, Sigma-Aldrich, St. Louis, USA) was prepared at a concentration of $0.200 \mathrm{~g} \mathrm{~L}^{-1}$. Working solutions used for the analytical curve were prepared in the linear range of the method. Sodium dodecyl sulfate was used as a model anionic surfactant and standard for construction of the analytical curve because the lauryl ether sulfate belonging to this class present a similar structure and the same reactivity as SDS. ${ }^{35}$ The reaction ratio between sodium dodecyl sulfate and methylene blue, and sodium lauryl ether sulfate (SLES) with methylene blue is the same (1:1 molar ratio), allowing the use of SDS as a model for the construction of analytical curves and the later detection of SLES in commercial products. In addition, the determination of the anionic surfactant (SDS or SLES) is performed by the indirect detection of residual methylene blue (MB) in $665 \mathrm{~nm}$ or using the red channel, so the ratio of the ionic pair formed is important (SDS-MB or SLES-MB).

A stock solution of methylene blue $(99.9 \%$, Merck, Darmstadt, Germany) was prepared at a concentration of $0.0300 \mathrm{~g} \mathrm{~L}^{-1}$, for use in the formation of the ionic pair in solution.

\section{Preparation of samples and analytical applications}

Two raw material samples of sodium lauryl ether sulfate $(27 \% \mathrm{~m} / \mathrm{m})$, two samples of liquid soap, and two samples 
of shower gel containing SLES were used to evaluate the performance of the proposed method. All the samples were obtained locally in the city of Araraquara (São Paulo State, Brazil), except for one raw material sample purchased in the city of São Paulo (São Paulo State, Brazil). An aliquot of the sample was weighted, slowly diluted in deionized water, and homogenized.

\section{Experimental design}

The ionic pair extraction parameters were evaluated using experimental design methodology. The effects of temperature $\left(25.0-60.0{ }^{\circ} \mathrm{C}\right.$ ), medium $\mathrm{pH}$ (acidic to basic, $3.0-11.0)$, solution ionic strength $\left(0.025-2.0 \mathrm{~mol} \mathrm{~L}^{-1}\right)$, and volume of reagents were studied one at a time. A central composite design was used to optimize the foam length (1.5-6.0 cm, maintaining the height and width constant) and the reaction time (60.0-180.0 $\mathrm{min})$.

Statistical analyses were performed using the Statistica 8.0 software package. ${ }^{39}$

\section{Construction of analytical curves}

Two analytical curves were constructed, one for detection by spectrophotometry and another for detection by digital imaging (Figure 1) using the working solution prepared by dilution of the stock solution with deionized water, as described below.

Recommended procedure: $5 \mathrm{~mL}$ of methylene blue stock solution $\left(0.0300 \mathrm{~g} \mathrm{~L}^{-1}\right)$ and $25.00 \mathrm{~mL}$ of the anionic surfactant working solution $\left(0.0100 \mathrm{~g} \mathrm{~L}^{-1}\right.$ to $\left.0.100 \mathrm{~g} \mathrm{~L}^{-1}\right)$ were transferred to a $50 \mathrm{~mL}$ beaker. A piece of polyurethane foam D28 $(1.00 \times 2.50 \times 3.75 \mathrm{~cm}$, mass $0.216 \pm 0.003 \mathrm{~g})$ with a magnetic stirring bar inside was added to the beaker. For each extraction a new PUF was used. The system was kept under stirring for $120 \mathrm{~min}$ at room temperature $\left(25-30{ }^{\circ} \mathrm{C}\right)$. After the agitation, the PUF containing the ionic pair was removed from the solution using tweezers to avoid contamination. The excess of methylene blue present in the solution not extracted by the PUF was used to indirectly quantify the anionic surfactant by the blue intensity decreasing.

\section{Spectrophotometry}

In the spectrophotometric method, the absorbance of the MB solution was measured at $665 \mathrm{~nm}$, before and after extraction of the ionic pair, with deionized water used as the blank. The analytical curve was constructed using the intensity of the MB solution absorption signal, before and after addition and extraction of increasing quantities of SDS (10.0-100.0 $\mathrm{mg} \mathrm{L}^{-1}$ ), with formation of the MB-SDS ionic pair on the surface of the PUF.

\section{Digital images}

For the digital image procedure, $600 \mu \mathrm{L}$ aliquots of the same solution used in the spectrophotometric measurements were added to the wells of the porcelain plate, which was placed in the homemade box for acquisition of images using a common cell phone equipped with a flash lamp. Identification of the most sensitive red, green and blue (RGB) channel was performed using ImageJ software, ${ }^{38}$ with determination of the effective intensity values. The experimental conditions for obtaining the images were the same for all the samples.

\section{Study of interferences}

Sulfate, chloride, and nitrate are reported in the official method as potential interferents in the reaction between anionic surfactants and cationic dyes. The official method states that the presence of these anions causes increased transfer of the ionic pair to the organic phase, due to the greater ionic strength. ${ }^{27}$ Furthermore, high concentrations of sulfate can react with $\mathrm{MB}$, forming a second ionic pair that can be transferred to the extraction phase, consequently decreasing the intensity of the MB in solution.

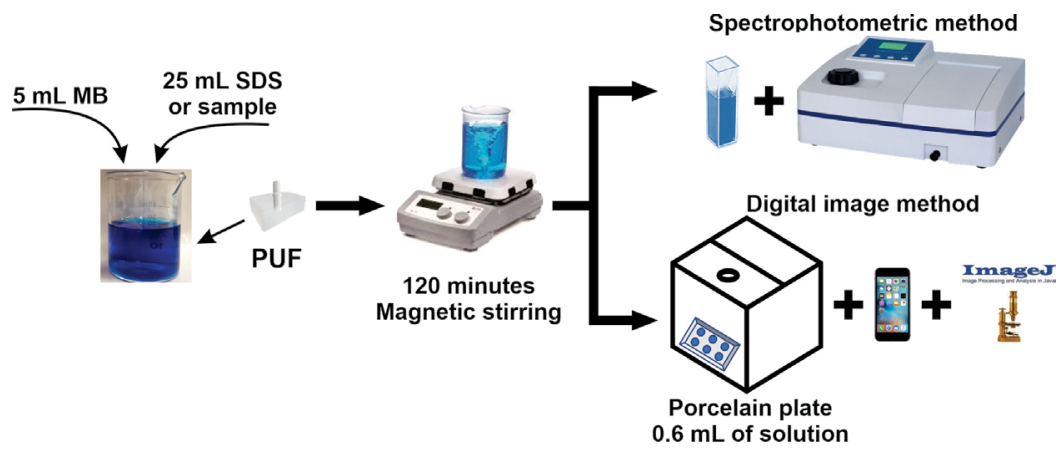

Figure 1. Scheme of the procedure for determination of total anionic surfactants in commercial samples, using PUF extraction followed by digital imaging or spectrophotometric analysis. 
Therefore, salts of these anions at concentrations of $0.025,0.050,0.250,0.500,1.00$, and $2.00 \mathrm{~mol} \mathrm{~L}^{-1}$ were used in the study of possible interferences.

Matrix interferences in the proposed method were evaluated using addition and recovery assays, with the addition of standards to the samples. For this, commercial samples containing $0.0250 \mathrm{~g} \mathrm{~L}^{-1}$ of SLES were spiked with SDS standards at values of $50 \%\left(0.0125 \mathrm{~g} \mathrm{~L}^{-1}\right)$, $100 \%\left(0.0250 \mathrm{~g} \mathrm{~L}^{-1}\right), 150 \%\left(0.0375 \mathrm{~g} \mathrm{~L}^{-1}\right)$, and $200 \%$ $\left(0.0500 \mathrm{~g} \mathrm{~L}^{-1}\right)$.

\section{Reference method}

Statistical comparison was made of the results obtained by the proposed method and the official spectrophotometric method for the determination of anionic surfactants. ${ }^{27}$ In the official method, the ionic pair formed by the reaction between an anionic surfactant and a cationic dye is extracted from an aqueous phase to an organic phase (chloroform PA, Qhemis, Jundiaí, Brazil), with the colored product being measured at $652 \mathrm{~nm}$.

\section{Results and Discussion}

Interaction between the methylene blue cationic dye and sodium dodecyl sulfate results in the formation of an ionic pair that has high affinity for polyurethane foam. The chemical structure of polyurethane foam is composed of two groups, polyol and diisocyanate. The polyurethane foam acts as a hydrophobic polymeric extractor which can interact with the non-polar segments on the ionic pair that is a longchain carbon of surfactant. The hydrophobic characteristics of the ionic pair decrease its affinity for the aqueous phase, increasing the interaction with the hydrophobic chain of the extractor phase and being removed by the polyurethane foam. ${ }^{40,41}$ The interaction is driven by the solution ionic strength and is more efficient in a neutral medium $(\mathrm{pH}=7$ was used in the developed method), since at acidic medium the hydrogen ions in solution act to ionize the $\mathrm{MB}$, while at basic medium there is competition between SDS and $\mathrm{OH}^{-}$ for the active sites. The sorption of the ionic pair formed in solution onto the polyurethane foam is mainly due to van der Waals forces. The interactions are shown in detail in Figure 2, based on reactions suggested in the literature. .0,41 $^{40}$

Preliminary experiments showed that the absorption of the ionic pair by the foam did not provide reproducible measurements, due to non-homogeneous sorption on the surface of the PUF, which affected the reproducibility of direct measurements by digital imaging of the surface of the foam. For this reason, it was decided to perform the digital image measurements using the solutions.

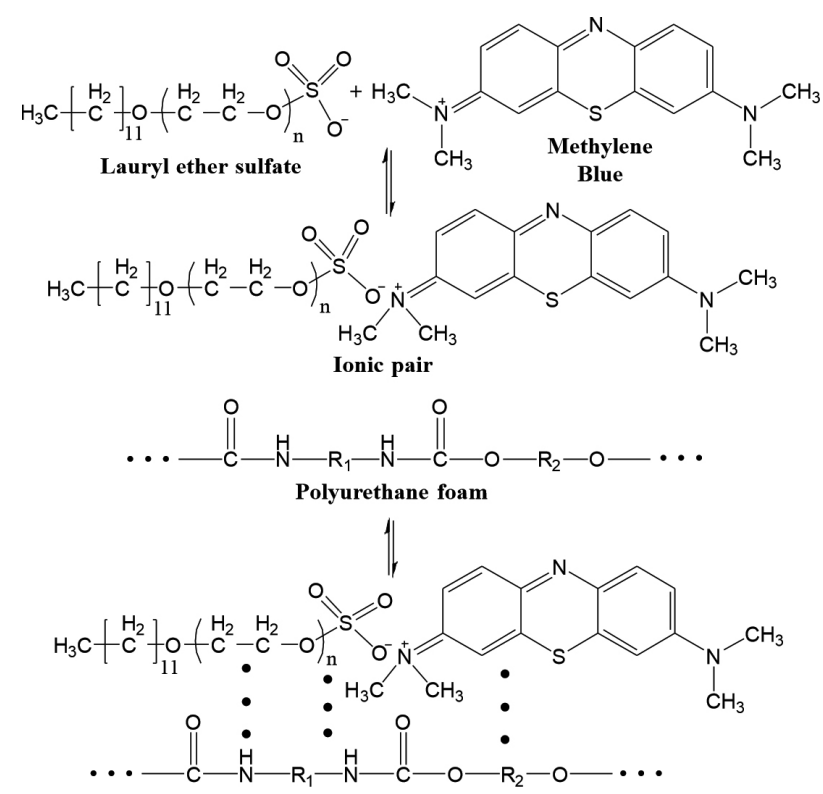

Figure 2. Scheme of the chemical equilibria involved in sorption of the ionic pair by the polyurethane foam.

In order to maximize extraction of the ionic pair, the extraction time and size of the polyurethane foam were optimized using a central composite design, for the two proposed methods.

\section{Optimization of variables}

For a complete and detailed study of the proposed methodology, evaluation was made of the effects of $\mathrm{pH}$, temperature, ionic strength, and volume of reagents. These were uncorrelated variables that had different effects on formation of the ionic pair, so individual optimizations were performed using a wide range of values for each variable (Table S1, Supplementary Information (SI) section). All the experiments were performed in triplicate, maintaining constant the concentrations of MB $\left(30.0 \mathrm{mg} \mathrm{L}^{-1}\right)$ and SDS (10.0 mg L $\mathrm{m}^{-1}$ ).

The results showed that the optimized conditions for extraction of the ionic pair from solution were temperature in the range $25-30{ }^{\circ} \mathrm{C}, \mathrm{pH}=7, \mathrm{MB}$ volume of $5.00 \mathrm{~mL}$, and SDS volume of $25.00 \mathrm{~mL}$. The ionic strength of the medium had no influence at salts concentrations below $0.500 \mathrm{~mol} \mathrm{~L}^{-1}$, so no alteration of the reaction solution was required, since the salts concentrations in the working solutions were below $0.050 \mathrm{~mol} \mathrm{~L}^{-1}$.

After these optimizations, a central composite design was performed, using the same concentrations of reagents and with the foam length and reaction time (magnetic stirring time) as correlated variables that could be adjusted to maximize the extraction efficiency. 

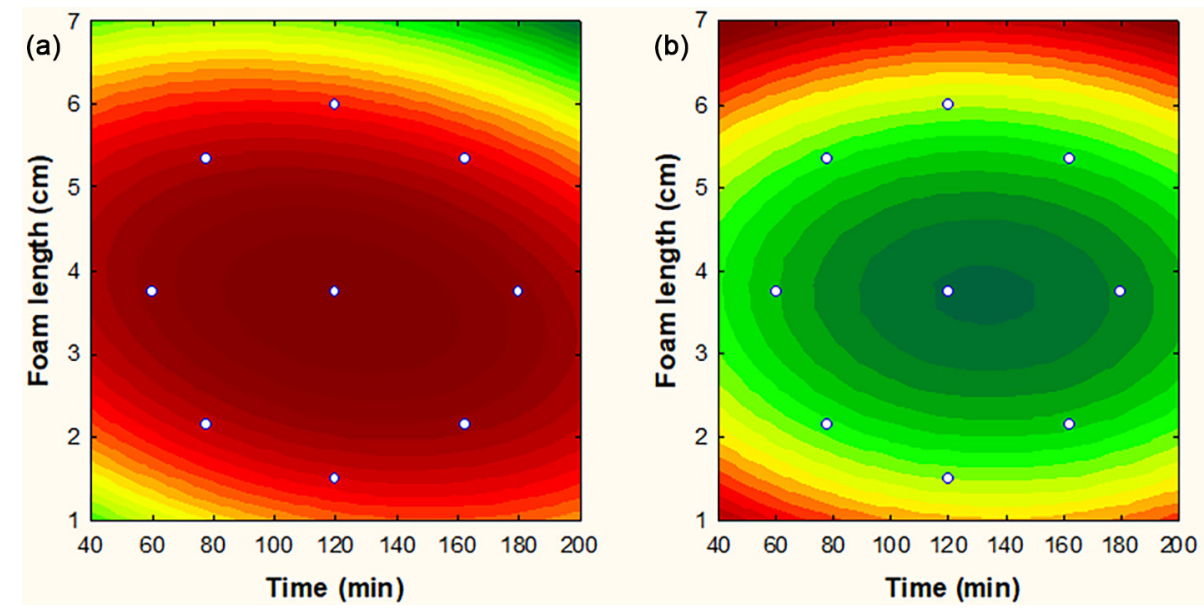

Figure 3. Central composite design response surfaces and their projections obtained for (a) effective intensity using digital imaging and (b) absorbance using spectrophotometry, as a function of foam length and reaction time.

\section{Optimization using central composite design}

After the optimization to obtain maximum formation of the ionic pair in solution, a central composite design was performed to optimize the values of the variables for extraction of the ionic pair to the polyurethane foam, in order to obtain the best possible analytical result. The composite design matrix for the extraction process is shown in Table S2 (SI section), where the combinations of the selected factors resulted in eleven experiments, carried out in triplicate, to identify the influence and significance of each factor. In this procedure, 5 levels were established for each variable: high $(-1.41$ and +1.41$)$, intermediate $(+1$ and -1$)$, and a central point $(0)$ in triplicate. In all the experiments, the MB and SDS concentration were maintained constant at 100.0 and $30.0 \mathrm{mg} \mathrm{L}^{-1}$, respectively. The data shown in Table S2 (SI section) were used to obtain the central composite design response surfaces and projections for the digital imaging (Figure 3a) and spectrophotometric (Figure 3b) methods, enabling identification of the best analytical conditions.

A statistically significant quadratic model accounting for $89.0 \%$ of the variance was fitted to the data, describing the relation between the maximum absorbance (spectrophotometric method) or effective intensity in the red channel (digital imaging method) and the optimal experimental conditions. Equations 1 and 2 provide the quadratic regression models for the spectrophotometric and digital imaging methods, respectively.

$$
\begin{aligned}
& Z=1.0912-0.00440 x+0.0000156 x^{2}-0.272 y+ \\
& 0.0356 y^{2}+0.0000676 x y
\end{aligned}
$$

where, $\mathrm{Z}$ is the response factor corresponding to the absorbance value, and $x$ and $y$ are the magnetic stirring time and foam length, respectively. The optimized parameter values for the spectrophotometric method were a stirring time of $120 \mathrm{~min}$ and foam length of $3.75 \mathrm{~cm}$.

$\mathrm{Z}=-0.644+0.00592 x-0.0000182 x^{2}+0.339 y-$ $0.0401 y^{2}-0.000416 x y$

where, $\mathrm{Z}$ is the response factor corresponding to the effective intensity in the red channel, and $x$ and $y$ are the magnetic stirring time and foam length, respectively. The optimized parameter values for the digital imaging method were a stirring time of $120 \mathrm{~min}$ and foam length of $3.75 \mathrm{~cm}$.

\section{Analytical features}

After optimization of the variables, an analytical curve was constructed for the anionic surfactant in the concentration range from 10.0 to $100 \mathrm{mg} \mathrm{L}^{-1}\left(3.46 \times 10^{-5}\right.$ to $\left.3.46 \times 10^{-4} \mathrm{~mol} \mathrm{~L}^{-1}\right)$, by appropriate dilution of a stock standard solution of SDS. The range of the analytical curve was determined according to the maximum extraction of the ionic pair in solution, maintaining linearity.

The analytical curve was made in triplicate, for each extraction was used a PUF of the same size and mass, and three stirrers of the same brand. The extraction procedures could be done in batch, decreasing the required time to perform multiple analysis. For analysis of the samples, the analytical curve was repeated, and the analysis of three samples were made in triplicate in one day. The total time was $120 \mathrm{~min}$.

Validation of the proposed methods considered the following analytical parameters: linear range, precision, accuracy, limit of detection (LOD), and limit of quantification (LOQ). Repeatability assays employed intra- and inter-day analytical curves, which resulted in 
relative standard deviation (\%RSD) values below $10 \%$ for all concentrations of the analytical curves and both quantification methods.

The LOD and LOQ values were determined according to the International Union of Pure and Applied Chemistry (IUPAC) recommendations, ${ }^{42}$ with a calculation based on 3.3 and 10 times the ratio between the standard deviation of the blank and the slope estimated from the calibration curve of the analyte. The parameters are shown in Table 1, with LOD and LOQ values of 2.71 and $9.28 \mathrm{mg} \mathrm{L}^{-1}$ $\left(9.40 \times 10^{-6}\right.$ and $\left.3.21 \times 10^{-5} \mathrm{~mol} \mathrm{~L}^{-1}\right)$, respectively, for the digital imaging method, and 1.74 and $5.83 \mathrm{mg} \mathrm{L}^{-1}$ $\left(6.03 \times 10^{-6}\right.$ and $\left.2.02 \times 10^{-5} \mathrm{~mol} \mathrm{~L}^{-1}\right)$, respectively, for the spectrophotometric method.

Table 1. Analytical features of the spectrophotometry and digital imaging methods

\begin{tabular}{lcc}
\hline \multirow{2}{*}{ Parameter } & \multicolumn{2}{c}{ Value } \\
\cline { 2 - 3 } & Spectrophotometry & Digital imaging \\
\hline Linear range / $\left(\mathrm{mg} \mathrm{L}^{-1}\right)$ & $10.0-100$ & $10.0-100$ \\
$\mathrm{R}^{2}$ & 0.993 & 0.991 \\
LOD & 1.74 & 2.71 \\
LOQ & 5.83 & 9.28
\end{tabular}

Analytical curve $\quad \mathrm{A}^{\mathrm{a}}=0.94-0.0062 \mathrm{C} \quad \mathrm{I}_{\mathrm{e}}^{\mathrm{b}}=0.0044+0.0025 \mathrm{C}$

${ }^{\mathrm{a}} \mathrm{A}$ : absorbance at $665 \mathrm{~nm}$; ${ }^{\mathrm{b}} \mathrm{e}$ : effective intensity; C: concentration of the analyte; $\mathrm{R}^{2}$ : coefficient of determination; LOD: limit of detection; LOQ: limit of quantification

\section{Study of interferences}

The presence of anions would alter the ionic strength in the aqueous solution containing the ionic pairs, increasing their transfer to the polyurethane foam by the saltingout effect. ${ }^{34}$ It is consistent with a solvent extraction mechanism, as an ion with a large charge density should be more strongly solvated reducing the number of solvent molecules available to solvate the organic compound, which has its solubility decreased and would therefore be force out of the solvent phase into the foam. Therefore, evaluation was made of the effects of the anion's chloride, sulfate, and nitrate at concentrations between 0.025 and $2.0 \mathrm{~mol} \mathrm{~L}^{-1}$. Especial attention was given to the effect of sulfate at concentrations higher than $0.50 \mathrm{~mol} \mathrm{~L}^{-1}(1.00$ and $2.00 \mathrm{~mol} \mathrm{~L}^{-1}$ were tested), since this anion can react with MB to produce a second ionic pair that could be transferred to the polyurethane foam. Subsequently, the experimental conditions were all adjusted to values below $0.050 \mathrm{~mol} \mathrm{~L}^{-1}$, at which there were no significant differences between the results for the two methods, with no alteration of transfer of the ionic pair to the polyurethane foam.

\section{Application to personal care hygiene products and recovery} study

The efficiencies of the methods were evaluated by their use to determine sodium lauryl ether sulfate, an anionic surfactant widely used in commercial products. Table 2 summarizes the results obtained for the six samples containing SLES, using the two proposed methods and the official method. The results (Table 2) showed that there was consistency among the concentrations found by the proposed spectrophotometric and digital imaging methods, and the official method. The $t$-values obtained were lower than the tabulated value, indicating no statistically significant differences between the methods.

A recovery study was used to investigate the accuracies of the proposed methods and the presence of matrix interferences. The SLES samples were spiked at concentrations 50,100,150, and $200 \%$ higher than the concentration found in the determination step. The spiked samples were analyzed using addition of SDS standards, with each analysis carried out in triplicate. The results (Table 3 ) showed that both quantification methods provided excellent accuracy (96.8-103\%) for the determination of SLES in the samples, without any matrix effects and with low RSD values. The results obtained by the proposed methods were confirmed by analyzing the samples using the official method.

Table 2. Determination of SLES in samples of raw material, shower gel, and liquid soap by the proposed and comparative methods

\begin{tabular}{lccccc}
\hline Sample & Official method / $(\%, \mathrm{~m} / \mathrm{m})$ & Spectrophotometry / $(\%, \mathrm{~m} / \mathrm{m})$ & $t$-test $^{\mathrm{a}}$ & Digital imaging / $(\%, \mathrm{~m} / \mathrm{m})^{t \text {-test }}$ \\
\hline $\mathrm{A}^{\mathrm{b}}$ & $26.0 \pm 0.2$ & $25.8 \pm 0.4$ & 0.41 & $26.0 \pm 0.1$ & 0.37 \\
$\mathrm{~B}^{\mathrm{b}}$ & $26.3 \pm 0.2$ & $26.2 \pm 0.3$ & 0.39 & $26.1 \pm 0.2$ & 1.18 \\
$\mathrm{C}^{\mathrm{c}}$ & $32.1 \pm 0.2$ & $32.3 \pm 0.2$ & 1.18 & $32.0 \pm 0.3$ & 0.92 \\
$\mathrm{D}^{\mathrm{c}}$ & $30.4 \pm 0.3$ & $30.0 \pm 0.6$ & 1.03 & $30.1 \pm 0.5$ & 0.89 \\
$\mathrm{E}^{\mathrm{c}}$ & $33.6 \pm 0.2$ & $33.5 \pm 0.2$ & 0.51 & $33.5 \pm 0.3$ & 0.77 \\
$\mathrm{~F}^{\mathrm{c}}$ & $33.1 \pm 0.2$ & $33.1 \pm 0.1$ & 0.42 & $32.8 \pm 0.4$ & 1.11 \\
\hline
\end{tabular}

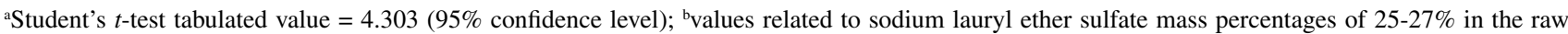
material samples; ${ }^{c}$ values related to mass percentages of $25-27 \%$ of the sodium lauryl ether sulfate standard in the samples. 
Table 3. Recovery data for SLES in samples of commercial shower gel and liquid soap, using the spectrophotometry and digital imaging analysis methods

\begin{tabular}{|c|c|c|c|c|c|}
\hline \multirow{2}{*}{ Sample } & \multirow{2}{*}{ Added / $\left(\mathrm{mg} \mathrm{L}^{-1}\right)$} & \multicolumn{2}{|c|}{ Spectrophotometry } & \multicolumn{2}{|c|}{ Digital imaging } \\
\hline & & Found / $\left(\mathrm{mg} \mathrm{L}^{-1}\right)$ & Recovery / \% & Found / $\left(\mathrm{mg} \mathrm{L}^{-1}\right)$ & Recovery / \% \\
\hline \multirow{6}{*}{$\mathrm{A}^{\mathrm{a}}$} & - & $25.0^{\mathrm{b}}$ & - & $25.0^{\mathrm{b}}$ & - \\
\hline & 12.5 & 38.5 & 103 & 38.3 & 102 \\
\hline & 25.0 & 50.7 & 101 & 50.3 & 101 \\
\hline & 37.5 & 62.6 & 100 & 63.4 & 101 \\
\hline & 50.0 & 74.5 & 99.4 & 74.2 & 99.0 \\
\hline & & & $\mu^{\mathrm{c}}=101 \pm 1$ & & $\mu^{\mathrm{c}}=101 \pm 1$ \\
\hline \multirow{6}{*}{$\mathrm{B}^{\mathrm{d}}$} & - & $25.0^{\mathrm{b}}$ & - & $25.0^{\mathrm{b}}$ & - \\
\hline & 12.5 & 36.5 & 97.4 & 36.3 & 96.8 \\
\hline & 25.0 & 49.1 & 98.4 & 49.0 & 98.1 \\
\hline & 37.5 & 62.4 & 100 & 62.4 & 99.9 \\
\hline & 50.0 & 74.1 & 98.8 & 74.0 & 98.6 \\
\hline & & & $\mu^{\mathrm{c}}=98.7 \pm 1.2$ & & $\mu^{\mathrm{c}}=98.4 \pm 1.3$ \\
\hline
\end{tabular}

${ }^{\mathrm{a}}$ Shower gel sample; ${ }^{\mathrm{b}}$ quantification of SLES in commercial samples; ${ }^{\mathrm{c}}$ average \pm relative standard deviation (RSD) of three determinations; ${ }^{\mathrm{d} l i q u i d}$ soap sample.

Therefore, the methods developed in this study constitute valuable tools for the determination of SLES in commercial samples, offering fast analysis, simplicity, and low cost. The methods are environmentally friendly, avoiding the use of organic solvents.

\section{Reuse of the polyurethane foam}

Investigation was made of the ability to reuse the same PUF in new extraction processes, after cleaning it in ethanolic medium to remove the ionic pairs adsorbed in the previous extraction. Good results were obtained reusing the PUF in up to 6 extraction processes, demonstrating an additional advantage of the technique, in addition to its simplicity, speed, and low cost.

\section{Conclusions}

Solvent-free and reliable alternative methods were successfully developed for the analysis of anionic surfactants (such as SLES) in samples of commercial personal care and hygiene products. The methods presented satisfactory sensitivity for determination of the analyte in commercial matrices, with the advantage of being environmentally friendly and not requiring elaborate cleanup steps or other sample treatments.

The commercial polyurethane foam showed high affinity for the ionic pair, with the same foam being able to be reused in up to six successive extractions, without any loss of efficiency. In addition, the method with digital imaging detection presented higher precision values, since the measurement was performed using a solution with high uniformity, in contrast to other reported methods employing reflectometric measurement on the PUF. ${ }^{35,36}$

The proposed methods therefore constitute valuable tools that contribute to filling a gap in the literature regarding the analysis of anionic surfactants in personal care products and cosmetics, without requiring use of the chlorinated solvent employed in the official method.

\section{Supplementary Information}

Supplementary information (Tables S1-S2) is available free of charge at http://jbcs.sbq.org.br as PDF file.

\section{Acknowledgments}

The authors would like to thank the Brazilian agencies CAPES (Finance Code 001), FAPESP (grant No. 2015/21733-1), and CNPq (grant No. 306661/2017-9) for financial support.

\section{References}

1. Rosen, M. J.; Kunjappu, J. T.; Surfactants Interfacial Phenom, $4^{\text {th }}$ ed.; John Wiley and Sons: New York, 2012.

2. Attwood, D.; Florence, A. T.; Surfactant Systems, Their Chemistry, Pharmacy and Biology, $1^{\text {st }}$ ed.; Chapman and Hall: London, 1983.

3. Kumar, D.; Rub, M. A.; J. Mol. Liq. 2018, 250, 329.

4. Azum, N.; Rub, M. A.; Asiri, A. M.; Colloids Surf., B 2014, 121,158

5. Tripathi, S. K.; Tyagi, R.; Nandi, B. K.; J. Dispersion Sci. Technol. 2013, 34, 1526. 
6. Atkins, P. W.; Loretta, J.; Princípios de Química: Questionando a Vida Moderna e o Meio Ambiente, $3^{\text {rd }}$ ed.; Bookman: Porto Alegre, 2006.

7. Adamson, A. W.; Gast, A. P.; Physical Chemistry of Surfaces, $6^{\text {th }}$ ed.; John Wiley and Sons: New York, 1997.

8. Mulqueen, P.; Adv. Colloid Interface Sci. 2003, 106, 83.

9. Könnecker, G.; Regelmann, J.; Belanger, S.; Gamon, K.; Sedlak, R.; Ecotoxicol. Environ. Saf. 2011, 74, 1445.

10. Holmberg, K.; Jönsson, B.; Kronberg, B.; Lindman, B.; Surfactants and Polymers in Aqueous Solution, $2^{\text {nd }}$ ed.; John Wiley and Sons: England, 2002.

11. Behera, P. K.; Mohapatra, S.; Patel, S.; Mishra, B. K.; J. Photochem. Photobiol., A 2005, 169, 253.

12. Myers, D.; Surfactant Science and Technology, $3^{\text {rd }}$ ed.; John Wiley and Sons: New Jersey, 2006.

13. Verge, C.; Moreno, A.; Bravo, J.; Berna, J. L.; Chemosphere 2001, 44, 1749.

14. Venhuis, S. H.; Mehrvar, M.; Int. J. Photoenergy 2004, 6, 115.

15. Ivanković, T.; Hrenović, J.; Arh. Hig. Rada Toksikol. 2010, 61, 95.

16. Boonyasuwat, S.; Chavadej, S.; Malakul, P.; Scamehorn, J. F.; Chem. Eng. J. 2003, 93, 241.

17. Lee, J.; Jing, J.; Yi, T. P.; Bose, R. J. C.; Mccarthy, J. R.; Tharmalingam, N.; Madheswaran, T.; Int. J. Environ. Res. Public Health 2020, 17, 3326.

18. Reid, V. W.; Longman, G. F.; Heinerth, E.; Tenside 1967, 4, 292.

19. Khamis, M.; Bulos, B.; Jumean, F.; Manassra, A.; Dakiky, M.; Dyes Pigm. 2005, 66, 179.

20. Jurado, E.; Fernández-Serrano, M.; Núñez-Olea, J.; Luzón, G.; Lechuga, M.; Chemosphere 2006, 65, 278.

21. Li, S.; Zhao, S.; Anal. Chim. Acta 2004, 501, 99.

22. Portet, F. I.; Treiner, C.; Desbène, P. L.; J. Chromatogr. A 2000, $878,99$.

23. Paun, I.; Iancu, V. I.; Cruceru, L.; Niculescu, M.; Chiriac, F. L.; Rev. Chim. 2018, 69, 27.
24. Tanaka, T.; Hiiro, K.; Kawahara, A.; Anal. Lett. 1974, 7, 173.

25. Gerlache, M.; Sentürk, Z.; Viré, J. C.; Kauffmann, J. M.; Anal. Chim. Acta 1997, 349, 59.

26. Sànchez, J.; Del Valle, M.; Electroanalysis 2001, 13, 471.

27. ISO 7875/1: Water Quality-Determination of SurfactantsPart 1: Determination of Anionic Surfactants by the Methylene Blue Spectrometric Method, ISO: Geneva, 1984.

28. Anastas, P. T.; Crit. Rev. Anal. Chem. 1999, 29, 167.

29. Keith, L. H.; Gron, L. U.; Young, J. L.; Chem. Rev. 2007, 107, 2695.

30. de La Guardia, M.; Garrigues, S.; Challenges in Green Analytical Chemistry, $1^{\text {st }}$ ed.; Royal Society of Chemistry: Cambridge, United Kingdom, 2011.

31. Bowen, H. J. M.; J. Chem. Soc. A 1970, 7, 1082.

32. Farag, A. B.; Ei-Wakil, A. M.; Ei-Shahawi, M. S.; Mashaly, M.; Anal. Sci. 1989, 5, 415.

33. El-Shahawi, M. S.; Chromatographia 1993, 36, 318.

34. Mori, M.; Cassella, R. J.; Quim. Nova 2009, 32, 2039.

35. Feiteira, F. N.; dos Reis, L. G. T.; Pacheco, W. F.; Cassella, R. J.; Microchem. J. 2015, 119, 44.

36. Yeerum, C.; Wongwilai, W.; Grudpan, K.; Vongboot, M.; Talanta 2018, 190, 85.

37. Salvador, A.; Chisvert, A.; Analysis of Cosmetic Products, $1^{\text {st }}$ ed.; Elsevier Science: Amsterdam, 2007.

38. Rasband, W. S.; ImageJ, version 1.51w; U. S. National Institutes of Health, Bethesda, Maryland, USA, 2018.

39. Statistica, version 8.0; Stat Soft Inc., Tulsa, OK, USA, 2007.

40. Sultan, M.; Environ. Chem. Lett. 2017, 15, 347.

41. Baldez, E. E.; Robaina, N. F.; Cassella, R. J.; J. Hazard. Mater. 2008, $159,580$.

42. Thompson, M.; Ellison, S. L. R.; Wood, R.; Pure Appl. Chem. 2002, 74, 835
Submitted: September 13, 2021

Published online: December 15, 2021 